\title{
What is Biliary Hyperkinesia?
}

\author{
James J Greenberg* \\ Mercy Walworth Hospital and Medical Center, Lake Geneva, WI, USA
}

\begin{abstract}
Background: What is biliary hyperkinesia? In a patient with typical symptoms consistent with biliary colic (postprandial right upper quadrant abdominal pain, nausea, bloating), what does a provider do when no gallstones are seen on routine imaging? Nuclear medicine imaging (CCK-HIDA scan) is often ordered as a next step. What does one do if the results come back with a markedly elevated ejection fraction after CCK administration?
\end{abstract}

Methods: Retrospective chart review.

Results: Thirteen patients presented with this proposed diagnosis and underwent laparoscopic cholecystectomy with resolution of symptoms in all.

Conclusions: Biliary hyperkinesia is a relatively infrequently used term to describe a subset of patients with no gallstones and an abnormally functioning gallbladder. This entity may be successfully treated with cholecystectomy.

\section{Introduction}

What do you do when a patient presents to you with biliary type symptoms and an elevated gallbladder ejection fraction (EF)? Not just an EF in normal or high normal range, but an extremely high off the charts number. The following article presents and discusses 13 patients with a markedly elevated EF, typical biliary presentation, and underwent a laparoscopic cholecystectomy with complete resolution of their symptoms. Herein, a diagnosis of biliary hyperkinesia is proposed to explain elevated ejection fractions in patients with typical biliary colic symptoms.

\section{Methods}

This is a retrospective chart review of 13 patients presenting with typical biliary colic symptoms who have undergone extensive preoperative work ups including ultrasound and CCK-HIDA scan in all, as well as, upper endoscopy and CT imaging in some (patient preference in those not having EGD/CT).

\section{Results/Patients}

1. 51-year-old female EF 97\% (path chronic cholecystitis)

2. 72-year-old female EF 99\% (path chronic cholecystitis)

3. 12-year-old female EF 97\% (path chronic cholecystitis)

4. 36-year-old female EF 85\% (path normal gallbladder)

5. 49-year-old female EF $88 \%$ (path chronic cholecystitis)

6. 16-year-old female EF $96 \%$ (path chronic cholecystitis)

7. 21-year-old male EF 99\% (path chronic cholecystitis)

8. 75-year-old female EF $85 \%$ (path chronic cholecystitis)

9. 63-year-old male EF $83 \%$ (path chronic cholecystitis)

10. 44-year-old male EF $91 \%$ (path chronic cholecystitis)

11. 48-year-old female EF $71 \%$ (path chronic cholecystitis)
12. 40-year-old female EF $98 \%$ (path normal gallbladder)

13. 32-year-old female EF $88 \%$ (path chronic cholecystitis)

Summary of above results: 13 total patients, average EF $90.5 \%$ (range 71\%-99\%). 10 females and 3 males. Age range 12 years to 75 years. All but two had "chronic cholecystitis" on pathology. All have had complete symptom relief (range 3 years for patient \#3 to 3 months for patient \#13). All but two had reproduction of their preoperative symptoms with CCK-HIDA (patient \#10, patient \#8).

\section{Summary}

As general surgeons, we are asked to see patients presenting with abdominal pain on a regular basis. The evaluation of a patient with typical gallbladder/biliary colic symptoms (post prandial right upper quadrant abdominal pain, nausea, bloating) and gallstones requiring cholecystectomy is straight forward. Most often, this procedure can be performed via a minimally invasive approach with good results. A patient with biliary type symptoms and a low EF (ideally, with reproduction of their symptoms after CCK infusion) also commonly undergo cholecystectomy. This latter group is termed biliary dyskinesia. A small percentage (30\%) will not have their symptoms completely relieved after cholecystectomy [1]. For this reason, a complete work up should be done prior to laparoscopic cholecystectomy which will be discussed below. But, what to do with a patient with no stones and a markedly elevated EF? The literature is sparse on data regarding this patient population. The purpose of this report is to shed more light on this topic and hopefully provide relief to many patients struggling with biliary type symptoms and a negative work up. This by no means is a

Correspondence to: James J Greenberg, Mercy Walworth Hospital and Medical Center, Lake Geneva, WI, USA, Tel: 262-245-0535, (815) 943-5431; E-mail: jgreenberg@mhemail.org

Received: March 07, 2018; Accepted: March 14, 2018; Published: March 23 2018 
panacea for all unexplained GI complaints. Rather, in the appropriate patient presenting with typical symptoms, a markedly elevated EF, and, ideally, reproduction of these symptoms, a cholecystectomy may fix the problem so to speak. This article summarizes the results of 13 patients with what is termed "biliary hyperkinesia". All patients had markedly elevated EF's, typical biliary colic presentation, and complete relief of all of their preoperative symptoms after surgery. Of note, all patients had HIDA scans performed/interpreted by the same group of radiologists, using standardized formats. This is a short term follow up and patients have only been followed for 3 months to a little more than 3 years. One would think that recurrence or persistence of symptoms would have occurred by now. Furthermore, these patients were all cared for in a small rural community so retained follow up is near universal. Nearly all patients had evidence of chronic cholecystitis on final pathology, suggesting that this hyperkinetic process is damaging the gallbladder and thus causing symptoms.

The pathogenesis of abnormally functioning gallbladders is not clear. The common theory is that it represents a motility disorder, often part of a generalized abnormal GI process associated with impaired gastric emptying and abnormal colonic transit [2]. The physiology involved with biliary motility is dependent upon both hormonal factors (CCK) and anatomy (obstruction from ductal abnormalities or more commonly, stones). CCK is released from the duodenal lumen in response to fat and amino acids. Lower ejection fractions (termed biliary dyskinesia), in theory, is manifested by less than normal gallbladder emptying thus leading to biliary stasis and mucosal damage (chronic cholecystitis) [3]. Exact measurements of the amount of CCK released and the actual release of biliary contents from the gallbladder (ejection fraction) is difficult to quantify. And thus, a CCK-HIDA scan can only give an estimate of what those numbers truly are. It does make sense that a hypersensitivity, if you will, in response to CCK release, or increased CCK levels themselves, could lead to over-aggressive emptying of the gallbladder (elevated EF) and potential symptoms $[3,4]$. We hypothesize, that this increased emptying could in turn cause elevated biliary pressures in this otherwise low-pressure system and lead to mucosal damage (cholecystitis). This is a short and narrow "circuit", bordered by gallbladder/cystic duct junction above, and sphincter or Oddi below. In theory, elevated pressures in this confined space could lead to symptoms typical of biliary colic. Many (but not all) patients in our study and others have shown evidence of chronic cholecystitis on histological analysis. This also makes sense that such a higher than normal pressure environment would cause biliary symptoms. And, therefore, it makes further sense to remove the end organ (gallbladder) in order to alleviate these symptoms [4]. The unanswered question is why some patients with elevated EFs have symptoms and others do not. It is important to note symptom reproduction after CCK administration and thus the likelihood of success after cholecystectomy.

As mentioned above, a thorough work up should be done prior to recommending surgery [5]. In a typical biliary colic presentation, an ultrasound should be the starting point. If no stones or sludge is seen (many believe that sludge/gravel represents enough evidence for biliary/gallbladder stasis and would recommend cholecystectomy), then a HIDA scan is often ordered next (Figure 1). A low EF could imply biliary dyskinesia in which case surgery would be recommended. If a markedly elevated EF is encountered, this could represent an abnormal finding possibly amenable to surgical correction. Even with a markedly elevated EF on HIDA scan, all other avenues and possible explanations of a patient's pain should be exhausted first. This includes ruling out cardiac disease and acid peptic disease/gastroesophageal reflux. Prescribing a short course of acid lowering medicine to see if

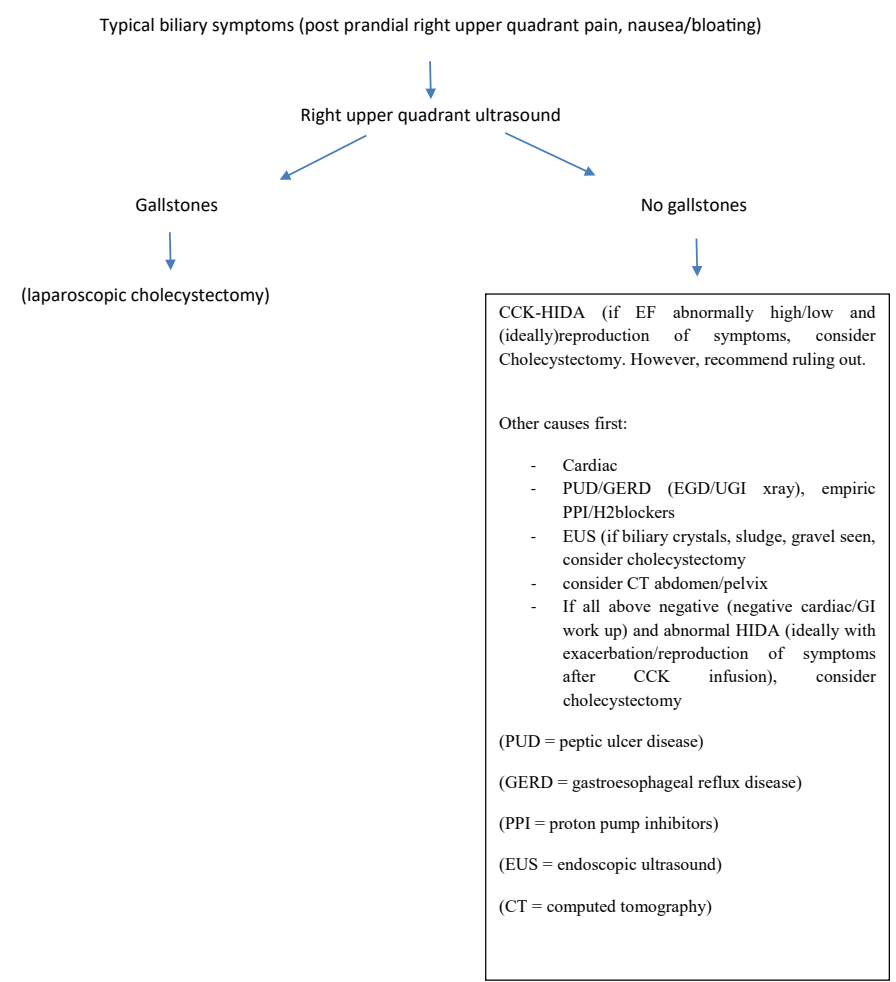

Figure 1. Flow Chart showing the diagnosis of biliary hyperkinesia

symptoms abate is in order. Moving on to imaging, a CT or UGI can be considered. Endoscopic ultrasound in search for biliary crystals should also be considered. If crystals are encountered, many would recommend cholecystectomy. EGD with bile aspiration, again looking for biliary microcrystals can be considered. Some also have talked about botulinum injection into the sphincter of Oddi to determine if they have sphincter dysfunction as a cause for their symptoms (if symptoms resolve after botox, then ERCP with sphincterotomy is recommended.) However, some authors have refuted this as a successful treatment option either before or after cholecystectomy [6].

It is not clear what "cut off" value should be used to place a patient in the biliary hyperkinesia category. Using $65 \%$ or greater as the cut off for biliary hyperkinesia, very few reports have been presented in the literature. It is not clear what value should be utilized to designate the term hyperkinesia. Cook, et al., first reported this in 1999, presenting seven patients with EF's greater than $84 \%$ and all had improvement after cholecystectomy [7]. Holes-Lewis presented 28 patients with EF's greater than $80 \%$ with all but one patient having improvement after cholecystectomy [8]. Steele et al., presented two patients, both with EFs greater than 90\%, who achieved symptom improvement after gallbladder removal [9]. And, Decoin noted success after cholecystectomy in 19 patients with an average EF of $75 \%$ and termed this "normokinetic biliary dyskinesia" [10].

It should be made clear to these patients preoperatively that not all patients will benefit from surgery with this diagnosis of biliary hyperkinesia. Laparoscopic cholecystectomy is not without complications and should not be entered into without considerable thought. Having said that, biliary hyperkinesia is a diagnosis that should be entered into the differential work up of a patient with biliary colic symptoms and can be cured with cholecystectomy. I invite others to report larger data subsets to further support or refute this diagnosis. 
The limitations of this study include its small sample size and short term follow up, as well as single center. Certainly, a "placebo" effect could be at play. However, with some of these patients having extended follow up with no return of their preoperative symptoms, this seems less likely. But, time will further clarify this. We hope to continue to accrue more patients over time and continue to submit our data. Our HIDA scans are standardized in terms of how they are performed. Furthermore, our pathology department has not submitted their slides for second opinion interpretations. However, they are "blinded" to the clinical information of each of these patients. So, in theory, they would not be "biased" in terms of reading "cholecystitis" unless otherwise warranted. The diagnosis of biliary dyskinesia, whether referring to low ejection fractions, or markedly elevated numbers as is discussed in this paper, is not a common diagnosis in our general surgical practice. More than 200 cholecystectomies are performed in our institution annually and dyskinesia represents about $10 \%$ of those gallbladders removed. There is not a clear explanation as to why these 13 patients have presented in such a fashion, though, it would seem that more will likely follow and allow for continued observation of this entity.

\section{Example algorithm}

A patient presents with typical biliary colic symptoms (postprandial right upper quadrant pain, nausea/bloating)- first order right upper quadrant ultrasound (if stones seen, proceed with cholecystectomy. If no stones, proceed with-CCK-HIDA scan (if abnormal low/high $\mathrm{EF}$ and ideally, reproduction of symptoms), consider cholecystectomy. However, recommend ruling out other causes first. This includes ruling out cardiac etiologies as well as peptic ulcer disease/gastroesophageal reflux disease (Esophagogastroduodenoscopy, UGI x-ray, endoscopic ultrasound) and/or empiric medication treatment with proton pump inhibitors/Histamine 2 blockers. If biliary crystals, sludge, gravel seen on endoscopy/ultrasound, consider cholecystectomy.

\section{Conclusion}

In conclusion, patient's presenting with typical biliary colic symptoms and a markedly elevated ejection fraction, termed biliary hyperkinesia, is an uncommon diagnosis, but may represent an entity that can be readily treated successfully with cholecystectomy. We look to continue to monitor this diagnosis and submit more data in the future.

\section{Disclosure}

No financial relationships exist with any pharmaceutical or device company. Dr Greenberg has nothing to disclose.

\section{References}

1. Singhal V, Szeto P, Norman H, Walsh N, Cagir B, et al. 2012 Biliary dyskinesia: how effective is cholecystectomy? J Gastrointest Surg 16: 135-140. [Crossref]

2. Penning C, Gielkens HA, Delemarre JB, Lamers CB, Masclee AA (1999) Gall bladder emptying in severe idiopathic constipation. Gut 45: 264-268. [Crossref]

3. Grace PA, Poston GJ, Williamson RCN (1990) Biliary Motility. Gut 31:571-82 [Crossref]

4. Huckaby L, Timmapuri S, Prasad R (2013) Biliary hyperkinesia: A potentially surgically correctable disorder in adolescents. $J$ of Pediatric Surgery Case Reports 1 : 314-16.

5. Cotton PB, Elta GH, Carter CR, Pasricha PJ, Corazziari ES (2016) Rome IV. Gallbladder and Sphincter of Oddi Disorders. Gastroenterology. [Crossref]

6. Cotton PB, Durkalski V, Romagnuola J, Pauls Q, Fogel E, et al. (2014) Effect of endoscopic sphincterotomy for suspected sphincter of Oddi dysfunction on pain related disabililty following cholecystectomy: the EPISOD randomized clinical trial. JAMA 311(20): 2101-9. [Crossref]

7. Cook $\mathrm{CH}$, Kisner J, Melvin WS, et al. Biliary hyperkinesia: a new indication for cholecystectomy. Society for Surgery of the Alimentary Tract: 1999

8. Holes-Lewis KA, Hakim S, Rehman F, Nabi H, Uhde T (2009) CCk-induced gallbladder hyperkinesia: An indication for cholecystectomy and brain-GI connectivity research. J Nucl Med. 50: (suppl 2): 1312.

9. Steele J, Wayne M, Iskander M, Wolmer T, Bratcher J (2014) Biliary pain, no gallstonesremove the gallbladder anyway? J Fam Pract 63 (8): 421-423. [Crossref]

10. Ducoin C, Faber R, Ilagan M, Ruderman W, Wier D (2012) Normokinetic biliary dyskinesia: a novel diagnosis. Surg Endosc. 26: 3088-3093. [Crossref]

Copyright: (C2018 Greenberg JJ. This is an open-access article distributed under the terms of the Creative Commons Attribution License, which permits unrestricted use, distribution, and reproduction in any medium, provided the original author and source are credited. 